\title{
A linear polar molecule in a two-color cw laser field: a symmetry analysis
}

\author{
David Mellado-Alcedo, ${ }^{1}$ Niurka R. Quintero, ${ }^{2}$ and Rosario González-Férez ${ }^{1}$ \\ ${ }^{1}$ Instituto Carlos I de Física Teórica y Computacional, \\ and Departamento de Física Atómica, Molecular y Nuclear, \\ Universidad de Granada, 18071 Granada, Spain \\ ${ }^{2}$ Departamento de Física Aplicada I, Universidad de Sevilla, 41011 Sevilla, Spain
}

(Dated: January 28, 2020)

\begin{abstract}
A theoretical study of the rotational dynamics of a linear polar molecule in a two-color non-resonant $\mathrm{cw}$ laser field is presented. By systematically considering the interactions of this field with the electric dipole moment, polarizability and hyperpolarizability of the molecule, the effect of the symmetries of the Hamiltonian on the orientation and alignment is explored in a regime where the time-average approximation does not hold. It is shown that the alignment and orientation satisfy certain symmetries as a function of the phases and field strengths. On average a one-color cw laser field doest not orient the molecule, being necessary a two-color one having odd and even products of the laser frequency to break the head-versus-tail order confinement.
\end{abstract}

\section{INTRODUCTION}

Biharmonic signals are widely used in many areas of physics in order to break the time-shift symmetry of the external forces and of the electromagnetic fields [1-6]. This symmetry breaking induces a plethora of unexpected phenomena, as for example, the dissipation-induced net motion, the current reversals by increasing the amplitudes, and resonances as a function of the frequency and of the damping coefficient $[7,8]$. These phenomena have been observed in seemingly unrelated systems, such as semiconductors [9], Josephson junctions [10], optical lattices [11], ferrofluids [12], Brownian particles [2], Bose-Einstein condensates [13], or solitons in non-linear systems [14-16]. Recent studies show that, regardless of the system, the symmetries of the biharmonic force determine the dependence of the measurements of the amplitudes and phases of this biharmonic force [17-19].

The spherical symmetry of a thermal sample of molecules is broken by inducing orientation and alignment [20-23] with experimental techniques such as brute force orientation [24-26], combined electrostatic and nonresonant laser fields [27-33], THz pulses [34-39], or the phase-locked two-color laser field [40-44]. An aligned molecule is characterized by the confinement of the molecular fixed axes along the laboratory fixed frame, and keeping the head-versus-tail symmetry. For an oriented molecule, this symmetry is broken and the dipole moment is pointing towards one hemisphere rather than the opposite. In the spirit of biharmonic signals, continuous-wave non-resonant laser fields could be employed to create directional states of polar molecules, rather than the laser pulses used in experiments, whose time-envelope is often given by a gaussian function.

Here, a linear polar molecule in a two-color continuouswave non-resonant laser field is considered. Within the Born-Oppenheimer and the rigid-rotor approximations, the field-dressed rotational dynamics is analyzed. The laser frequency is chosen so that the time-average approximation [45] is not correct, but still assuming that no electronic, vibrational or rotational transitions are driven by this field. By systematically including in the Hamiltonian the interactions of the field with the electric dipole moment, polarizability and hyperpolarizability, the effect of the symmetries of the system on the orientation and alignment is analyzed. Due to these symmetries, it is shown that it is not possible to orient on average the polar molecule with a one-color cw laser field being necessary to employ a two-color one, whose summed frequencies should be an odd multiple of the main laser frequency. In addition, the alignment and orientation satisfy certain symmetries with respect to the relative phase of the two electric field components, and can be expressed as series expansions for a fixed propagation time.

The paper is organized as follow. In Sec. II, the Hamiltonian of the system and its symmetries are described, and how they affect to the expectation values that characterize the field-dressed rotational dynamics. The results are analyzed and discussed in Sec. III. In Sec. III A the validity of the time-average approximation is investigated by including only the interaction between the two-color electric field and the molecular dipole moment, and if higher order terms in the interactions are taken into account. The results for the orientation and alignment are analyzed in Sec. III B and Sec. III C, respectively, by systematically including in the description the interactions of the electric field with the electric dipole moment, polarizability, and hyperpolarizability of the molecule. The conclusions are given in Sec. IV.

\section{THE SYSTEM AND THE HAMILTONIAN}

A linear polar molecule exposed to a phase-controlled continuous-wave (cw) two-color laser field linearly polarized along the laboratory fixed frame (LFF) $Z$-axis is considered. The corresponding electric field $\mathbf{E}(t)=E(t) \mathbf{Z}$ is given by the biharmonic function

$$
E(t)=\sum_{i=1,2} \epsilon_{i} \cos \left[q_{i} \omega\left(t+t_{0}\right)+\delta_{i}\right],
$$


with $q_{i} \omega, \epsilon_{i}$ and $\delta_{i}$ being the laser frequency, electric field strength and phase of the $i$-th harmonic, respectively, with $q_{i}$ being positive integers and $i=1,2 . t_{0}$ represents the time shift of the laser with respect to the initial time, i. e., when the molecule starts to interact with the two-color laser field, which was previously turned on, and, therefore $t_{0}$ might not be zero.

The molecule is described by the Born-Oppenheimer approximation, and the rotational motion is investigated using the rigid rotor approach. Within this framework, the Hamiltonian is given by $[46,47]$

$$
H=H_{0}+H_{\mu}+H_{\alpha}+H_{\beta},
$$

where the first term stands for the field-free Hamiltonian

$$
H_{0}=\mathrm{BJ}^{2},
$$

with $B$ being the rotational constant of the molecule, and $\mathbf{J}$ the rotational angular momentum operator. The second, third and fourth terms represent the interaction of the electric field with the electric dipole moment, polarizability, and hyperpolarizability of the molecule, respectively,

$$
\begin{aligned}
H_{\mu} & =-\mu \cos \theta E(t), \\
H_{\alpha} & =-\frac{1}{2}\left(\Delta \alpha \cos ^{2} \theta+\alpha_{\perp}\right) E^{2}(t), \\
H_{\beta} & =-\frac{1}{6}\left(\Delta \beta \cos ^{3} \theta+3 \beta_{\perp} \cos \theta\right) E^{3}(t) .
\end{aligned}
$$

In these expressions, $\theta$ is the Euler angle between the internuclear molecular axis and the LFF $Z$-axis, $\mu$ the permanent electric dipole moment, $\Delta \alpha=\alpha_{\|}-\alpha_{\perp}$ the polarizability anisotropy, with $\alpha_{\perp}$ and $\alpha_{\|}$being its perpendicular and parallel components, and $\Delta \beta=\beta_{\|}-3 \beta_{\perp}$ hyperpolarizability anisotropy, with $\beta_{\perp}$ and $\beta_{\|}$being the perpendicular and parallel components, respectively. The aim is to explore the dependence of the field-dressed rotational dynamics on the parameters of the two-color laser field, including its frequency. To do so, the laser frequency is reduced and considered within the regime where the time-average approximation is not correct [45], but still assuming that the laser electric field is non-resonant, i. e., it cannot drive any electronic, vibrational or rotational transition.

The Hamiltonian (2) is invariant under arbitrary rotations around the LFF $Z$-axis $\mathbf{C}_{\mathbf{Z}}(\epsilon)$, and reflections on the LFF $X Z$-plane, $\sigma_{\mathbf{X Z}}$. These symmetries imply that the eigenstates associated to Hamiltonian (2) with a constant electric field are degenerate in $|M|$, with $M$ being the projection of the rotational angular momentum along the LFF $Z$-axis. The time-dependent Schrödinger equation associated to the Hamiltonian (2) is solved by combining the short iterative Lanczos method [48] for the time variable, and a basis set expansion in terms of the field-free basis, formed by the spherical harmonics $Y_{J, M}(\Omega)$ with $\Omega=(\theta, \phi)$ being the Euler angles, including the symmetries of the Hamiltonian, i. e., fixed $M$. The time-dependent Schrödinger equation is solved assuming that at $t=0$ the molecule is in a field-free eigenstate, i. e., $\psi(\Omega, t=0)=Y_{J, M}(\Omega)$.

In this work, the field-dressed rotational dynamics is analyzed in terms of the expectation values

$$
\left\langle\cos ^{k} \theta\right\rangle=\int \psi^{*}(\Omega, t) \cos ^{k} \theta \psi(\Omega, t) d \Omega,
$$

with $\psi(\Omega, t)$ being the time-dependent wave function, and positive $k$. For the orientation and alignment, $k=1$ and $k=2$, respectively. The wave function $\psi(\Omega, t)$ and the expectation values depend on the laser field parameters (1), and, therefore, they are invariant under the same symmetry transformations as the Hamiltonian (2).

The two-color electric field (1) is invariant under the following transformation

$$
\mathcal{T}:\left(q_{1}, q_{2}, \omega\right) \rightarrow\left(\kappa q_{1}, \kappa q_{2}, \frac{\omega}{\kappa}\right) \quad \text { with } \quad \kappa \in \mathbb{Z}^{+},
$$

and, therefore, the Hamiltonian (2) is also invariant under this transformation. As a consequence, the orientation and alignment are also invariant under $\mathcal{T}$. Thus, the symmetry analysis can be restricted to $q_{1}$ and $q_{2}$ satisfying $\operatorname{gcd}\left(q_{1}, q_{2}\right)=1$. The symmetries in the phases $\delta_{1}$ and $\delta_{2}$ and amplitudes $\epsilon_{1}$ and $\epsilon_{2}$ of the two-color electric field (1), imply that

$$
\begin{aligned}
& \left\langle\cos ^{k} \theta\right\rangle\left(t, t_{0}, \epsilon_{1}, \epsilon_{2}, \omega, \delta_{1}, \delta_{2}\right)= \\
& \left\langle\cos ^{k} \theta\right\rangle\left(t, t_{0},(-1)^{n_{1}} \epsilon_{1},(-1)^{n_{2}} \epsilon_{2}, \omega, \delta_{1}+n_{1} \pi, \delta_{2}+n_{2} \pi\right),
\end{aligned}
$$

with $n_{1}$ and $n_{2}$ being integers, and $k \in \mathbb{Z}^{+}$. Note that this expression shows explicitly its dependence on $t, t_{0}, \epsilon_{1}, \epsilon_{2}, \omega, \delta_{1}$, and $\delta_{2}$ of this expectation value. The inversion of the electric field direction gives rise to the following invariance

$$
\begin{aligned}
& \left\langle\cos ^{k} \theta\right\rangle\left(t, t_{0}, \epsilon_{1}, \epsilon_{2}, \omega, \delta_{1}, \delta_{2}\right)= \\
& (-1)^{k}\left\langle\cos ^{k} \theta\right\rangle\left(t, t_{0},-\epsilon_{1},-\epsilon_{2}, \omega, \delta_{1}, \delta_{2}\right),
\end{aligned}
$$

with $k \in \mathbb{Z}^{+}$. The Hamiltonian (2) is invariant under a temporal shift of $t_{0}$ by changing correspondingly the phases $\delta_{1}$ and $\delta_{2}$, and this expectation value satisfies

$$
\begin{array}{cc}
\left\langle\cos ^{k} \theta\right\rangle\left(t, t_{0}, \epsilon_{1}, \epsilon_{2}, \omega, \delta_{1}, \delta_{2}\right)= & (11) \\
\left\langle\cos ^{k} \theta\right\rangle\left(t, t_{0}+\tau, \epsilon_{1}, \epsilon_{2}, \omega, \delta_{1}-q_{1} \omega \tau, \delta_{2}-q_{2} \omega \tau\right) & \forall \tau,
\end{array}
$$

$k \in \mathbb{Z}^{+}$. In contrast to the rest of the laser field parameters, the value of $t_{0}$ cannot be easily controlled in an experiment. Therefore, these expectation values are averaged over $t_{0}[19]$ as

$$
\left\langle\left\langle\cos ^{k} \theta\right\rangle\right\rangle=\frac{\omega}{2 \pi} \int_{0}^{\frac{2 \pi}{\omega}} d t_{0}\left\langle\cos ^{k} \theta\right\rangle \quad \text { with } \quad k \in \mathbb{Z}^{+},
$$

where the integral is restricted to an electric field period due to the $t_{0}$ periodicity of the electric field (1).

For a one-color electric field, i. e., $\epsilon_{1}=0$ or $\epsilon_{2}=0$, the Hamiltonian (2) fulfills the symmetry in $t_{0} H\left(\theta, t_{0}\right)=$ $H\left(\pi-\theta, t_{0}+\frac{\pi}{q_{i} \omega}\right)$, with $\epsilon_{i} \neq 0$ and $i=1$ or 2 , and the 
expectation values satisfy

$$
\left\langle\cos ^{k} \theta\right\rangle\left(t_{0}+\frac{\pi}{q_{i} \omega}\right)=(-1)^{k}\left\langle\cos ^{k} \theta\right\rangle\left(t_{0}\right),
$$

and, therefore

$$
\left\langle\left\langle\cos ^{k} \theta\right\rangle\right\rangle=\left[1+(-1)^{k}\right] \frac{q_{i} \omega}{2 \pi} \int_{0}^{\frac{\pi}{q_{i} \omega}} d t_{0}\left\langle\cos ^{k} \theta\right\rangle,
$$

where the dependence on the field parameters is omitted. For $k=1$, relation (14) indicates that on average the molecule is not oriented by a one-color laser field, regardless of its frequency, even if all three interactions are taken into account. Note that for a fixed $t_{0}$, the molecule is oriented, whereas for $t_{0}+\frac{\pi}{q_{i} \omega}$, it gets the same orientation but in the opposite direction as shown in (13). As a consequence, the $t_{0}$-averaged orientation becomes zero. For $k=2$, equation (14) implies the interval of integration in (12) can be reduced to $t_{0}$ to $0 \leq t_{0} \leq \frac{\pi}{q_{i} \omega}$.

From the symmetries in Eq. (9), the $t_{0}$-averaged expectation value satisfies

$$
\begin{aligned}
& \left\langle\left\langle\cos ^{k} \theta\right\rangle\right\rangle\left(t, \epsilon_{1}, \epsilon_{2}, \omega, \delta_{1}, \delta_{2}\right)= \\
& \left\langle\left\langle\cos ^{k} \theta\right\rangle\right\rangle\left(t,(-1)^{n_{1}} \epsilon_{1},(-1)^{n_{2}} \epsilon_{2}, \omega, \delta_{1}+n_{1} \pi, \delta_{2}+n_{2} \pi\right),
\end{aligned}
$$

with $n_{1}$ and $n_{2}$ being integers. The symmetry due to the inversion of the electric field direction (10) reads as

$$
\begin{aligned}
& \left\langle\left\langle\cos ^{k} \theta\right\rangle\right\rangle\left(t, \epsilon_{1}, \epsilon_{2}, \omega, \delta_{1}, \delta_{2}\right)= \\
& \quad(-1)^{k}\left\langle\left\langle\cos ^{k} \theta\right\rangle\right\rangle\left(t,-\epsilon_{1},-\epsilon_{2}, \omega, \delta_{1}, \delta_{2}\right) .
\end{aligned}
$$

The invariance on $t_{0}(11)$ gives rise to the following phaseshift symmetry

$$
\begin{aligned}
& \left\langle\left\langle\cos ^{k} \theta\right\rangle\right\rangle\left(t, \epsilon_{1}, \epsilon_{2}, \omega, \delta_{1}, \delta_{2}\right)= \\
& \quad\left\langle\left\langle\cos ^{k} \theta\right\rangle\right\rangle\left(t, \epsilon_{1}, \epsilon_{2}, \omega, \delta_{1}+q_{1} \Delta, \delta_{2}+q_{2} \Delta\right)
\end{aligned}
$$

with $\Delta$ being an arbitrary phase-shift.

The symmetries (15), (16) and (17) imply additional identities for the $t_{0}$-averaged expectation values. For $q_{1}$ and $q_{2}$ odd integers, it yields

$$
\left\langle\left\langle\cos ^{k} \theta\right\rangle\right\rangle\left(t, \epsilon_{1}, \epsilon_{2}, \omega, \delta_{1}, \delta_{2}\right)=0,
$$

with $k$ being an odd integer, which indicates the lack of orientation for $k=1$, and

$$
\begin{gathered}
\left\langle\left\langle\cos ^{k} \theta\right\rangle\right\rangle\left(t, \epsilon_{1}, \epsilon_{2}, \omega, \delta_{1}, \delta_{2}\right)= \\
\left\langle\left\langle\cos ^{k} \theta\right\rangle\right\rangle\left(t, \epsilon_{1}, \epsilon_{2}, \omega, \delta_{1}+n_{1} \frac{\pi}{2}, \delta_{2}+\left(2-(-1)^{\frac{q_{2}-q_{1}}{2}}\right) n_{1} \frac{\pi}{2}\right),
\end{gathered}
$$

with $n_{1}$ being an integer, and $k$ an even integer. Thus, in this case the molecule is aligned but not oriented. Whereas for $q_{1}$ odd and $q_{2}$ even, it holds

$$
\begin{gathered}
\left\langle\left\langle\cos ^{k} \theta\right\rangle\right\rangle\left(t, \epsilon_{1}, \epsilon_{2}, \omega, \delta_{1}, \delta_{2}\right)= \\
(-1)^{k\left(\frac{n_{1} q_{2}}{2}+n_{2} q_{1}\right)}\left\langle\left\langle\cos ^{k} \theta\right\rangle\right\rangle\left(t, \epsilon_{1}, \epsilon_{2}, \omega, \delta_{1}+n_{1} \frac{\pi}{2}, \delta_{2}+n_{2} \pi\right)
\end{gathered}
$$

with $n_{1}$ and $n_{2}$ being integers, and, $k \in \mathbb{Z}^{+}$. Hence, in the latter case the molecule is both oriented and aligned.

Due to the symmetries of the Hamiltonian, the $t_{0^{-}}$ averaged expectation values of the orientation and alignment can be expressed as a series expansion in terms of the amplitude and phase, i. e., $\epsilon_{j}$ and $\delta_{j}$, of the $j$-harmonics due to the two-color electric field (1) for fixed time $t$ [19]. The corresponding expansions (A6), (A7), (A8), and (A9) are derived in the Appendix.

\section{RESULTS}

The carbonyl sulfide molecule OCS serve as prototype for this study. For OCS [49], the rotational constant is $B=0.20286 \mathrm{~cm}^{-1}$, the rotational period $T_{\text {rot }}=82.2 \mathrm{ps}$, the permanent electric dipole $\mu=0.71 \mathrm{D}$, polarizability anisotropy and perpendicular term $\Delta \alpha=27.26$ a.u., and $\alpha_{\perp}=26.08$ a.u., respectively, hyperpolarizability anisotropy and perpendicular term $\Delta \beta=132.3$ a.u., and $\beta_{\perp}=-59.1$ a.u., respectively. For $t=0$, the OCS is assumed to be in its rotational ground state, i.e., $\psi(\Omega, t=0)=Y_{0,0}(\Omega)$. Although this work is restricted to the field-dressed rotational dynamics of the ground state, similar results are obtained for excited rotational states.

Since the $t_{0}$-averaged expectation values satisfy the phase-shift symmetry (17), the phase of the first harmonic is fixed to zero, $\delta_{1}=0$. The electric field strengths are taken as $\epsilon_{1}=(1-\gamma) E_{0}$ and $\epsilon_{2}=\gamma E_{0}$ with $0 \leq \gamma \leq 1$. The field strength is related to the laser field intensity as $E_{0}=\sqrt{\frac{2 I}{c \epsilon_{0}}}$, with $c$ being the speed of the light and $\epsilon_{0}$ the vacuum electric permittivity. The laser intensity is fixed to $I=5 \cdot 10^{11} \mathrm{~W} / \mathrm{cm}^{2}$, with the electric field strength being $E_{0} \approx 1.94 \cdot 10^{7} \mathrm{~V} / \mathrm{cm}$. Note that this strong laser intensity is routinely used in non-resonant ac laser pulses, whereas is larger than the experimentally available intensities of cw lasers [50]. However, such a large laser intensity provokes a moderate orientation and a strong alignment of the molecule, and these effects can be analyzed in terms of the field symmetries. The electric field frequencies are fixed to $q_{1}=1$ and $q_{2}=2$, which provoke both the orientation and alignment of the molecule.

\section{A. Validity of the time-average approximation}

Despite of not considering a laser pulse, this section is devoted to investigate the validity of the time-average approximation [45]. For a non-resonant two-color laser field, if the field frequencies, $\omega$ and $2 \omega$, are far from any molecular resonance and higher than the molecular rotational frequency, the Hamiltonian (2) is averaged over the rapid oscillations of the non-resonant laser field. Note that for a laser pulse, it is further assumed that the laser period is much shorter than the pulse duration. If the time-average approximation would be correct, then the 


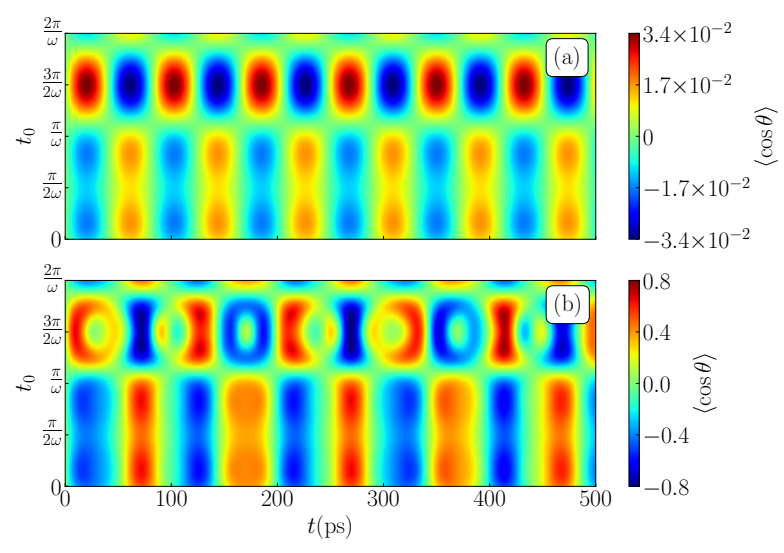

FIG. 1. For the Hamiltonian $H=H_{0}+H_{\mu}$, orientation versus time and $t_{0}$ for a two-color laser field with periods (a) $T=10 \mathrm{fs}$ and (b) $T=400 \mathrm{fs}$. The field parameters are fixed to $\gamma=0.5$, $\delta_{1}=0$, and $\delta_{2}=\pi / 2$.

Hamiltonian (2) would be reduced to

$$
\begin{aligned}
H & =B \mathbf{J}^{2}-\frac{1}{2}\left(\Delta \alpha \cos ^{2} \theta+\alpha_{\perp}\right) f_{1}\left(\epsilon_{1}, \epsilon_{2}, q_{1}, q_{2}, \delta_{1}, \delta_{2}\right) \\
& -\frac{1}{6}\left(\Delta \beta \cos ^{3} \theta+3 \beta_{\perp} \cos \theta\right) f_{2}\left(\epsilon_{1}, \epsilon_{2}, q_{1}, q_{2}, \delta_{1}, \delta_{2}\right)(21)
\end{aligned}
$$

with

$$
\begin{aligned}
f_{1}\left(\epsilon_{1}, \epsilon_{2}, q_{1}, q_{2}, \delta_{1}, \delta_{2}\right) & =\frac{\epsilon_{1}^{2}+\epsilon_{2}^{2}}{2}+\epsilon_{1} \epsilon_{2} \cos \left(\delta_{1}-\delta_{2}\right) \delta_{q_{1}, q_{2}} \\
f_{2}\left(\epsilon_{1}, \epsilon_{2}, q_{1}, q_{2}, \delta_{1}, \delta_{2}\right) & =\frac{3}{4} \epsilon_{1}^{2} \epsilon_{2} \delta_{2 q_{1}, q_{2}} \cos \left(2 \delta_{1}-\delta_{2}\right) \\
& +\frac{3}{4} \epsilon_{1} \epsilon_{2}^{2} \delta_{q_{1}, 2 q_{2}} \cos \left(\delta_{1}-2 \delta_{2}\right) .
\end{aligned}
$$

For a two-color electric field with $q_{1}=1$ and $q_{2}=2$, the time-averaged Hamiltonian (21) reads

$$
\begin{aligned}
H & =B \mathbf{J}^{2}-\frac{1}{4}\left(\Delta \alpha \cos ^{2} \theta+\alpha_{\perp}\right)\left(\epsilon_{1}^{2}+\epsilon_{2}^{2}\right) \\
& -\frac{1}{8}\left(\Delta \beta \cos ^{3} \theta+3 \beta_{\perp} \cos \theta\right) \epsilon_{1}^{2} \epsilon_{2} \cos \left(2 \delta_{1}-\delta_{2}\right) .
\end{aligned}
$$

Thus, depending on the values of the phases $\delta_{1}$ and $\delta_{2}$, this time-averaged Hamiltonian might align the molecule, or both orient and align it.

For a one-color laser field, the Hamiltonian (22) indicates that if the time-average approximation is correct the molecule should not be oriented. Regardless of the validity of the time-average approximation, the equality (14) shows that the $t_{0}$-averaged orientation is zero if only onecolor laser field is included in the full Hamiltonian (2). However, by fixing $t_{0}$ and considering only the interaction with the electric dipole moment, the absolute value of the orientation becomes larger than $10^{-2}$ for periods larger or equal than 10 fs and $|\langle\langle\cos \theta\rangle\rangle| \approx 10^{-8}$; whereas for $T=1 \mathrm{fs},|\langle\cos \theta\rangle| \approx 10^{-3}$ and $\langle\langle\cos \theta\rangle\rangle \approx 10^{-9}$. In the regime $T \gtrsim 10$ fs the time-average approximation starts to fail. The $t_{0}$-averaged alignment is non-zero, see Eq.
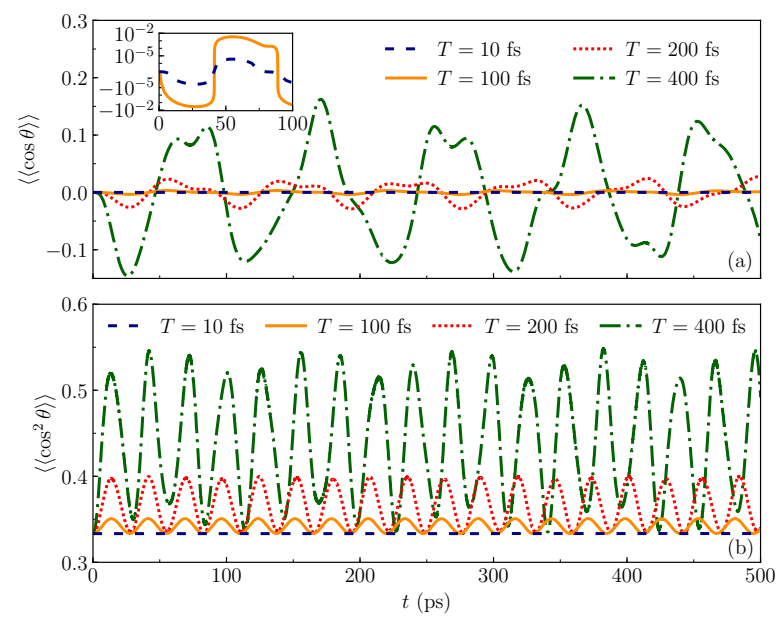

FIG. 2. For the Hamiltonian $H=H_{0}+H_{\mu}$, (a) orientation and (b) alignment averaged over $t_{0}$ as a function of time for the electric field periods $T=10 \mathrm{fs}$ (blue dashed line), $100 \mathrm{fs}$ (orange solid line), $200 \mathrm{fs}$ (red dotted line) and $400 \mathrm{fs}$ (green dot-dashed line). The electric field parameters are fixed to $\gamma=0.5, \delta_{1}=0$, and $\delta_{2}=\pi / 2$.

(14), and the maximal deviation from its field-free value, $\left\langle\left\langle\cos ^{2} \theta\right\rangle\right\rangle=1 / 3$, is of the order of $10^{-4}$. Whereas for a fixed $t_{0}$, the alignment deviation from its field-free value reaches up to $10^{-3}$ for a laser period of $10 \mathrm{fs}$. If the interactions of the electric field with the polarizability and hyperpolarizability are also included, the absolute value of the orientation is also greater than $10^{-2}$ for $T \gtrsim 10 \mathrm{fs}$ and a fixed $t_{0}$. The alignment for a fixed $t_{0}$ and the $t_{0}$-averaged alignment reach values up to 0.85 for a laser period of $10 \mathrm{fs}$.

For a two-color laser field, the simplest system including only the interaction of the field with the electric dipole moment, i. e., $H=H_{0}+H_{\mu}$ is first analyzed. Figs. 1 (a) and (b) show the orientation as a function of $t_{0}$ and time for laser field periods $10 \mathrm{fs}$ and $400 \mathrm{fs}$, respectively, and the field parameters $\gamma=0.5$ and $\delta_{2}=\pi / 2$. The orientation is non-zero even for the 10 fs laser period, and depends on $t_{0}$. These two features contradict the validity of the timeaverage approximation. Fig. 2 presents the $t_{0}$-averaged orientation and $t_{0}$-averaged alignment as a function of the time for the electric field periods $T=10,100,200$ and $400 \mathrm{fs}$, and $\gamma=0.5$ and $\delta_{2}=\pi / 2$. For $T=10 \mathrm{fs}$, the $t_{0}$-averaged orientation is of the order of $10^{-6}$. Note that $|\langle\cos \theta\rangle|$ is four orders of magnitude larger, but due to the dependence of $\langle\cos \theta\rangle$ on $t_{0}$, see Fig. 1 (a), the $t_{0}$-averaged $\langle\langle\cos \theta\rangle\rangle$ becomes very small. The maximal deviation of the $t_{0}$-averaged alignment from its field-free value is $1.8 \times 10^{-4}$. Thus, for $T=\frac{2 \pi}{\omega}=10 \mathrm{fs}$, on average the molecule is not oriented nor aligned. As a consequence, one can mistakenly conclude that the timeaverage approximation can be applied, however, for a fixed $t_{0}$, it is not correct as shown by the results in Fig. 1 (a). For $T=100 \mathrm{fs}$, the deviations of $\langle\langle\cos \theta\rangle\rangle$ and $\left\langle\left\langle\cos ^{2} \theta\right\rangle\right\rangle$ from the corresponding field-free values are still small 


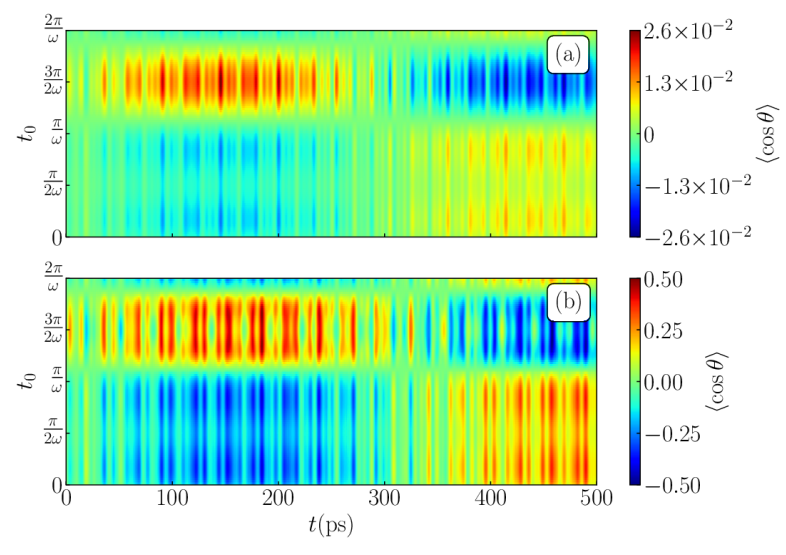

FIG. 3. For the full Hamiltonian $H=H_{0}+H_{\mu}+H_{\alpha}+H_{\beta}$, orientation versus time and $t_{0}$ for a two-color laser field with periods (a) $T=10 \mathrm{fs}$ and (b) $T=400 \mathrm{fs}$. The field parameters are fixed to $\gamma=0.5, \delta_{1}=0$, and $\delta_{2}=\pi / 2$.

but not negligible. By increasing the laser field period $T$, i.e., reducing the laser frequency $\omega$, the $t_{0}$-averaged orientation and $t_{0}$-averaged alignment increase, see for instance $\langle\langle\cos \theta\rangle\rangle$ and $\left\langle\left\langle\cos ^{2} \theta\right\rangle\right\rangle$ for $T=200$ and $400 \mathrm{fs}$.

For the three interactions, i. e., $H=H_{0}+H_{\mu}+H_{\alpha}+H_{\beta}$, Figs. 3 (a) and (b) show the orientation as a function of $t_{0}$ and time for the laser field periods $T=10 \mathrm{fs}$ and $400 \mathrm{fs}$, respectively, and the electric field parameters $\gamma=0.5$ and $\delta_{2}=\pi / 2$. As in the previous case, $\langle\cos \theta\rangle$ is non-zero and depends on $t_{0}$, which indicates that even for a $T=10 \mathrm{fs}$ laser, the time-average approximation is not correct. Fig. 4 shows the $t_{0}$-averaged orientation and $t_{0}$-averaged alignment as a function of the time for the electric field periods $T=10,100,200$ and $400 \mathrm{fs}$. Due to the dependence on $t_{0}$ of $\langle\cos \theta\rangle$, the $t_{0}$-averaged orientation is rather small even for the laser frequency $400 \mathrm{fs}$. This cancellation effect does not take place when $\left\langle\left\langle\cos ^{2} \theta\right\rangle\right\rangle$ is computed because $\left\langle\cos ^{2} \theta\right\rangle>0$. As a consequence, the $t_{0}$-averaged alignment is very large for all considered laser field periods.

\section{B. Orientation induced by the two-color laser field}

In this section, the rotational dynamics is explored, by first focusing on the orientation in a two-color laser field with period $T=\frac{2 \pi}{\omega}=400$ fs and $\delta_{1}=0$. Note that this electric field period is two orders of magnitude larger than the period of the non-resonant lasers used typically in experiment such as YAG-Laser and Ti-Shaphire [51]. However, such a large electric field period ensures a certain degree of the $t_{0}$-averaged orientation of the molecule, and allows us to analyze the field-dressed dynamics in terms of the field symmetries. This is done by systematically including in the description the interactions of electric field with the electric dipole moment, polarizability, and hyperpolarizability of the molecule.

By considering only the interaction of the electric field with its permanent electric dipole moment, i. e., $H=H_{0}+$
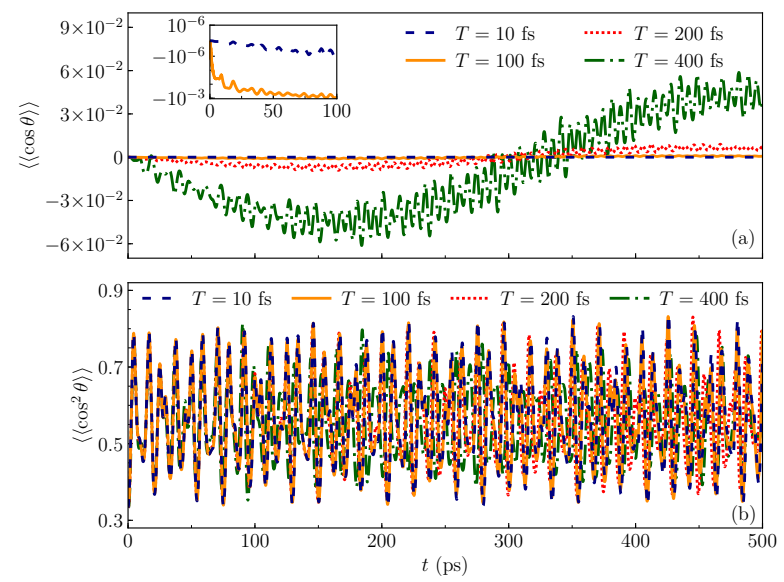

FIG. 4. For the three interactions, (a) orientation and (b) alignment averaged over $t_{0}$ versus time for the electric field periods $T=2 \pi / \omega=10 \mathrm{fs}$ (blue dashed line), $100 \mathrm{fs}$ (orange solid line), $200 \mathrm{fs}$ (red dotted line) and 400 fs (green dotdashed line). The two-color electric field parameters are fixed to $\gamma=0.5, \delta_{1}=0$ and $\delta_{2}=\pi / 2$.

$H_{\mu}$, the contour plots Fig. 5 (a), (b) and (c) present the $t_{0}$-averaged orientation as a function of the propagation time $t$ and the phase of the second-harmonic $\delta_{2}$ for the strength parameters $\gamma=0.25,0.5$ and 0.75 , respectively. For a fixed time, $\langle\langle\cos \theta\rangle\rangle$ satisfies the symmetry (20) for $k=1, n_{1}=0$, and $n_{2}=1$, and approximately fulfills the relation $\langle\langle\cos \theta\rangle\rangle\left(t, \delta_{2}\right) \approx\langle\langle\cos \theta\rangle\rangle\left(t, \pi-\delta_{2}\right)$. Regardless of the values of $\gamma$ and $t,\langle\langle\cos \theta\rangle\rangle$ shows the same dependence on $\delta_{2}$, reaching its maximal orientation in absolute value for $\delta_{2} \approx \pi / 2$ and $\delta_{2} \approx 3 \pi / 2$, and the minimal one for $\delta_{2} \approx 0$ and $\delta_{2} \approx \pi$. For a certain $\gamma$ and $\delta_{2},\langle\langle\cos \theta\rangle\rangle$ oscillates as a function of time, and the field-dressed wave function has contributions of only few field-free states. Note that for $\delta_{2} \approx 0, \pi$, the amplitude of these oscillations is very small.

By adding the interaction of the electric field with the molecular polarizability, i.e., $H=H_{0}+H_{\mu}+$ $H_{\alpha}$, the field-dressed dynamics becomes more complex. The corresponding $t_{0}$-averaged orientation is presented in Fig. 5 (d), (e) and (f) for $\gamma=0.25,0.5$ and 0.75, respectively. In this case, $\langle\langle\cos \theta\rangle\rangle$ also satisfies the symmetry relation (20) for $k=1, n_{1}=0$, and $n_{2}=1$. In addition, the dependence of $\langle\langle\cos \theta\rangle\rangle$ on the second-harmonic phase for fixed time $t$ strongly depends on the parameter $\gamma$, i.e., on the relative weight of the electric field components. For $\gamma=0.25$, the $t_{0}$-averaged orientation is composed of slow oscillations with superimposed fast modulations of the amplitude, and it is lower than 0.12 . In contrast, an orientation up to 0.24 is achieved for $\gamma=0.5$ and $\gamma=0.75$, and $\langle\langle\cos \theta\rangle\rangle$ slowly oscillates with time, whereas the amplitude also show small oscillations.

The $t_{0}$-averaged orientation when the three interactions are considered, i. e., $H=H_{0}+H_{\mu}+H_{\alpha}+H_{\beta}$, is presented in Fig. 5 (g), (h) and (i) for $\gamma=0.25,0.5$ and 0.75 , respectively. The rotational dynamics in this case shows 


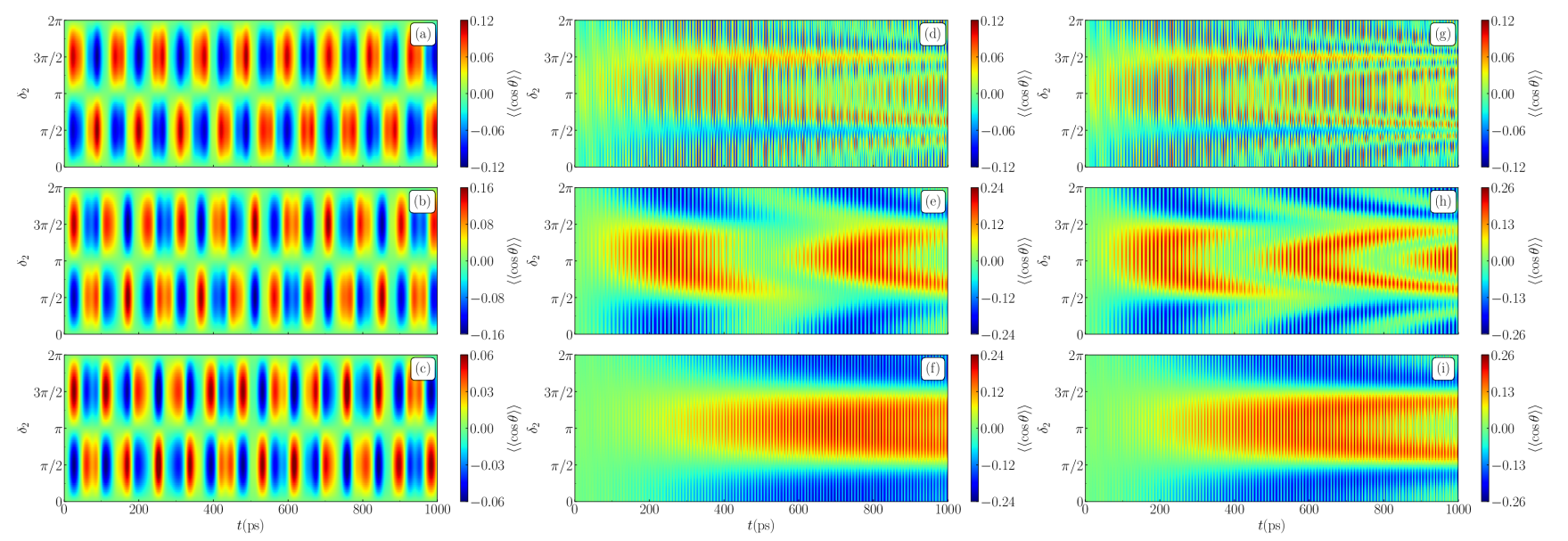

FIG. 5. Orientation averaged over $t_{0}$ as a function of the time and the second-harmonic phase for the parameters (a), (d) and (g) $\gamma=0.25$, (b), (e), and (h) $\gamma=0.5$ and (c), (f) and (i) $\gamma=0.75$. The interaction Hamiltonian includes $H_{\mu}$ in panels (a), (b) and (c); $H_{\mu}+H_{\alpha}$ in panels (d), (e) and (f); and $H_{\mu}+H_{\alpha}+H_{\beta}$ in panels (g), (h) and (i).

a qualitatively similar behavior as when $H_{\beta}$ is neglected, compare panels (d)-(g), (e)-(h) and (f)-(i) of Fig. 5. The absolute value of the orientation is slightly larger in this case, and for a fixed $\delta_{2}$, the oscillations as a function of $t$ show smaller periods.

For a fixed configuration of the two-color laser field and a certain propagation time $t$, the analytic expression (A8) of $\langle\langle\cos \theta\rangle\rangle$, and its dependence on the phase of the second harmonics $\delta_{2}$ is illustrated in Fig. 6. Panels (a) and (b) in Fig. 6 show the $t_{0}$-averaged orientation as a function $\delta_{2}$ for propagation times $t=200 \mathrm{ps}$ and $t=600 \mathrm{ps}$, respectively, and the two components of the electric field having the same weight, i. e., $\gamma=0.5$. These curves have been numerically fitted to the expansion (A8) using the $\delta_{2}$-independent constants $\mathcal{C}_{j}\left(t, \epsilon_{1}, \epsilon_{2}\right)$ and $\varphi_{j}\left(t, \epsilon_{1}, \epsilon_{2}\right)$ as fitting parameters with $j$ being an odd integer. Figs. 6 (c) and (d) show these fitted coefficients $\mathcal{C}_{j}\left(t, \epsilon_{1}, \epsilon_{2}\right)$ at $t=200 \mathrm{ps}$ and $t=600 \mathrm{ps}$, respectively, the fitted phases are shown in Figs. 6 (e) and (f). If only the interaction with the electric dipole moment is included, the first coefficient $j=1$ is sufficient to reproduce the dependence of $\langle\langle\cos \theta\rangle\rangle$ in $\delta_{2}$ with a fairly good accuracy, and the phase of this $j=1$ coefficient is close to $\pi / 2$, in agreement with the observed sine-like behaviour, i. e., $\langle\langle\cos \theta\rangle\rangle\left(t, \delta_{2}\right) \approx\langle\langle\cos \theta\rangle\rangle\left(t, \pi-\delta_{2}\right)$. Note that the next term in the expansion (A8) with $j=3$ is smaller than $5 \times 10^{-5}$ for these two propagation times. The deviation of the phases $\varphi_{1}\left(t, \epsilon_{1}, \epsilon_{2}\right)$ and $\varphi_{3}\left(t, \epsilon_{1}, \epsilon_{2}\right)$ from being exactly $\pi / 2$ prevents the $t_{0}$-averaged orientation from being exactly zero at $\delta_{2}=0$ and $\pi$. By adding the interaction with the molecular polarizability, higher order terms becomes more important in Eq. (A8). Indeed, the $j \geq 7(j \geq 11)$ coefficients are smaller than $10^{-4}$ for $t=200 \mathrm{ps}(t=600 \mathrm{ps})$. Finally, if the three interactions are considered, i. e., $H=H_{0}+H_{\mu}+H_{\alpha}+H_{\beta}$, the dependence of $\langle\langle\cos \theta\rangle\rangle$ in $\delta_{2}$ gets more complicated, and the contribution of higher order terms increases gaining importance on the expansion (A8). In these two cases, the fitted phases take values very close to $\pi$ or $2 \pi$, see Figs. 6 (e) and (f), and the deviation from these values prevents $\langle\langle\cos \theta\rangle\rangle$ from being zero at $\delta_{2}=\pi / 2$ and $3 \pi / 2$.

This analysis is completed by investigating the dependence of the $t_{0}$-averaged orientation on the relative weight of the two electric field components $\gamma$ in Fig. 7. When only the interaction with the electric dipole moment is taken into account, the results for $\delta_{2}=0, \pi / 2$, and $3 \pi / 4$ are presented in Fig. 7 (a), (b) and (c), respectively. As discussed in Sec. III A, the $t_{0}$-averaged orientation is zero for $\gamma=0$ and $\gamma=1$ because the electric field (1) becomes one-color. For fixed $\gamma$ and $\delta_{2},\langle\langle\cos \theta\rangle\rangle$ shows fast oscillation versus $t$. The dependence of $\langle\langle\cos \theta\rangle\rangle$ on $\gamma$ changes as the propagation time $t$ increases. The orientation tends to reach larger values for $0.25 \lesssim \gamma \lesssim 0.75$. The maximal orientation is 0.16 , which is achieved for $\delta_{2}=\pi / 2$. The orientation for $\delta_{2}=0$ is non zero but lower than $10^{-3}$.

For $H=H_{0}+H_{\mu}+H_{\alpha}$, Figs. 7 (d), (e) and (f) present $\langle\langle\cos \theta\rangle\rangle$ for $\delta_{2}=0, \pi / 2$ and $3 \pi / 4$, respectively. Compared to the previous case, the dependence of $\langle\langle\cos \theta\rangle\rangle$ on $\gamma$ and $t$ is significantly changed. As a function of $t,\langle\langle\cos \theta\rangle\rangle$ shows slow oscillations, whose amplitude is modulated. A significant orientation of the molecule is attained for several values of $\gamma$. The maximal absolute value of the orientation is reached for $\delta_{2}=0, \pi$ (not shown here), and $3 \pi / 4$, whereas the minimal one occurs for $\delta_{2}=\pi / 2$. Indeed, for $\delta_{2}=\pi / 2,|\langle\langle\cos \theta\rangle\rangle|$ is smaller than 0.08 , and it reaches close to 0.3 for $\delta_{2}=3 \pi / 4$ and 0 , respectively. By adding the interaction with the hyperpolarizability, i.e., $H=H_{0}+H_{\mu}+H_{\alpha}+H_{\beta}$, the $t_{0}$-averaged orientation is not significantly modified, see Fig. 7 (g), (h) and (i). In particular, $\langle\langle\cos \theta\rangle\rangle$ shows a qualitatively similar dependence on $t$ and $\gamma$ as in the previous case. 

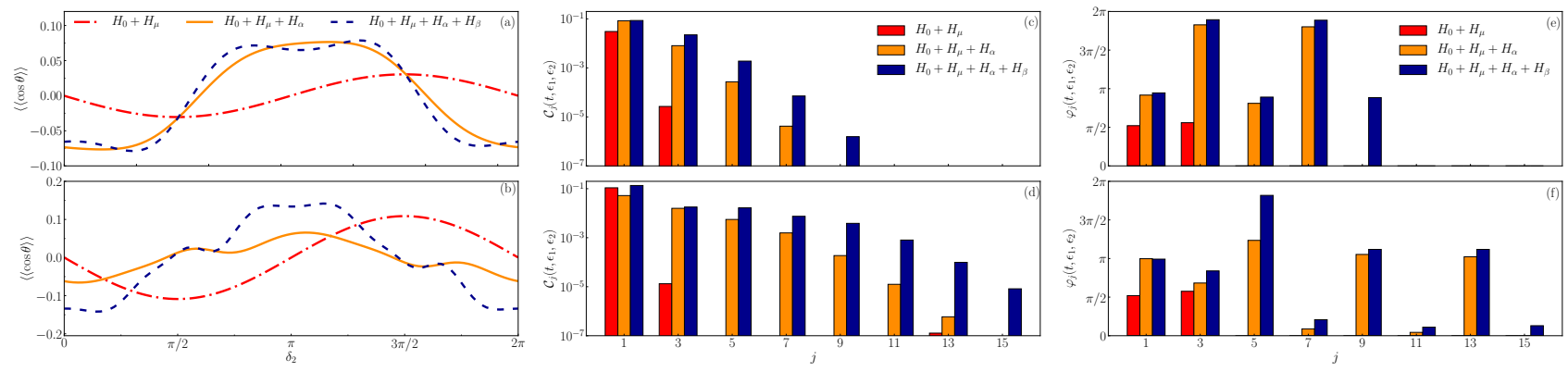

FIG. 6. For a two-color electric field with $\gamma=0.5, t_{0}$-averaged orientation as a function of the second-harmonic phase at fixed propagation times (a) $t=200 \mathrm{ps}$ and (b) $t=600 \mathrm{ps}$. (c) and (d) fitted coefficients $\mathcal{C}_{j}\left(t, \epsilon_{1}, \epsilon_{2}\right.$ ), and (e) and (f) fitted phases $\varphi_{j}\left(t, \epsilon_{1}, \epsilon_{2}\right)$ from the analytic expression (A8) of $\langle\langle\cos \theta\rangle\rangle$ for $t=200 \mathrm{ps}$ and $t=600 \mathrm{ps}$, respectively. The interaction Hamiltonian includes $H_{\mu}$ (red dot-dashed line), $H_{\mu}+H_{\alpha}$ (orange solid line), and $H_{\mu}+H_{\alpha}+H_{\beta}$ (blue dashed line).
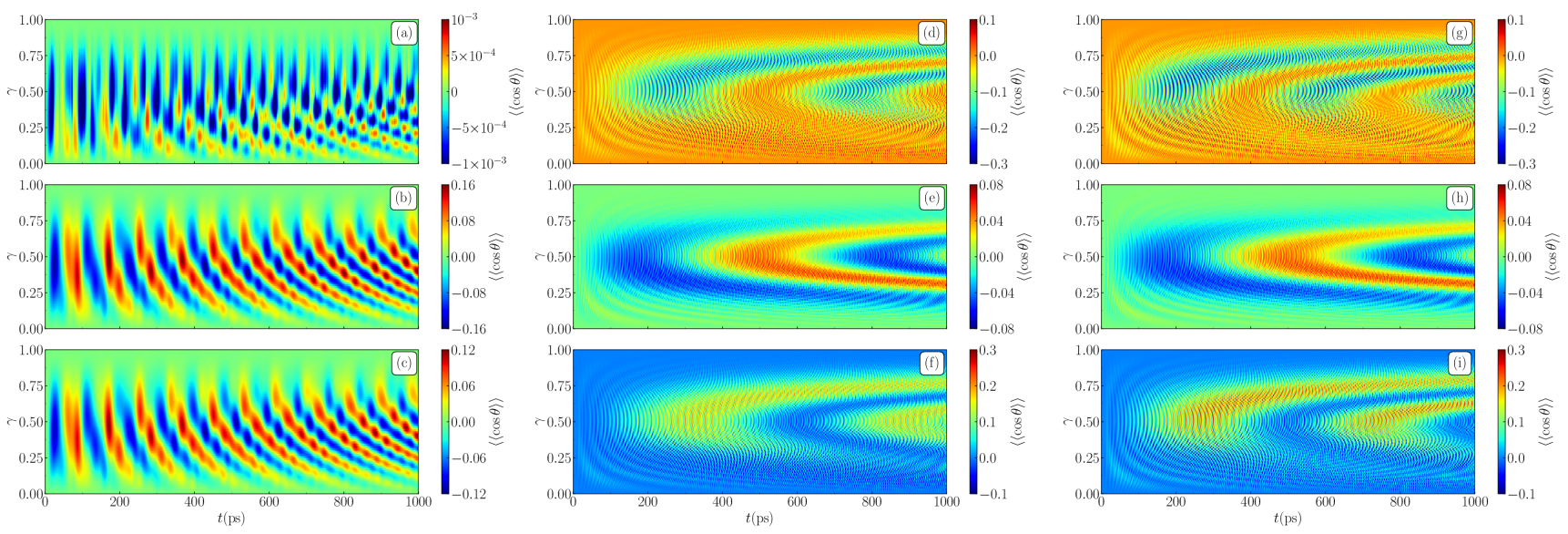

FIG. 7. The $t_{0}$-averaged orientation as a function of the propagation time and of the relative weight of the two electric field components $\gamma$ for the phase of the second-harmonic (a), (d), and (g) $\delta_{2}=0$; (b), (e) and (h) $\delta_{2}=\pi / 2$; and (c), (f) and (i) $\delta_{2}=3 \pi / 4$. The interaction Hamiltonian includes $H_{\mu}$ in panels (a), (b) and (c); $H_{\mu}+H_{\alpha}$ in panels (d), (e) and (f); and $H_{\mu}+H_{\alpha}+H_{\beta}$ in panels (g), (h) and (i). 

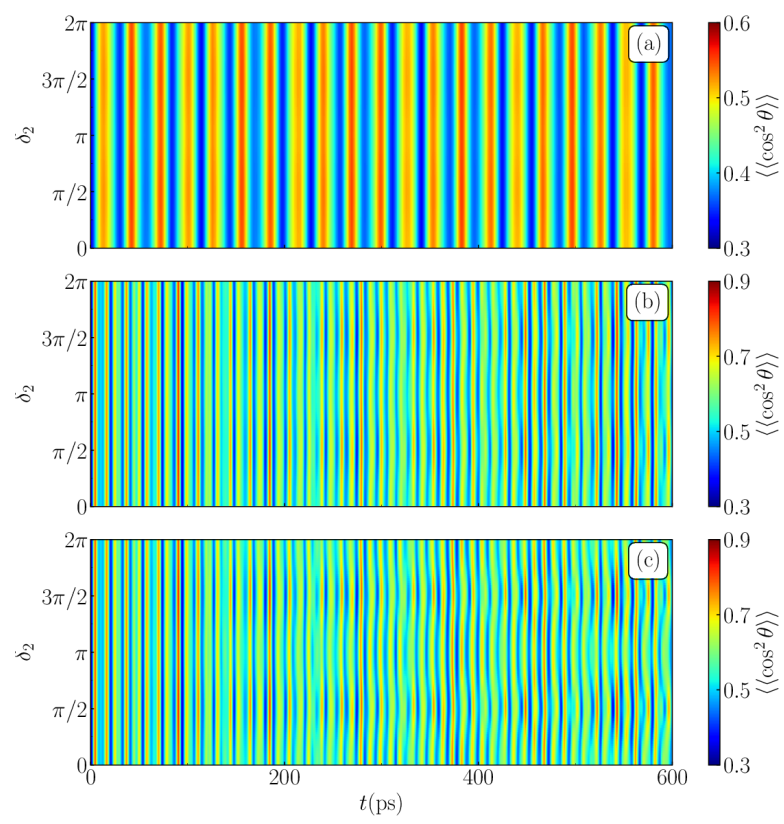

FIG. 8. The $t_{0}$-averaged alignment a function of the propagation time and the second-harmonic phase $\delta_{2}$ for the relative weight of the electric field components $\gamma=0.5$. The interaction Hamiltonian includes (a) $H_{\mu}$, (b) $H_{\mu}+H_{\alpha}$, and (c) $H_{\mu}+H_{\alpha}+H_{\beta}$.

\section{Alignment induced by the two-color laser field}

This section presents the $t_{0}$-averaged alignment of the molecule in a two-color laser field with period $T=400 \mathrm{fs}$. The panels (a), (b) and (c) of Fig. 8 present $\left\langle\left\langle\cos ^{2} \theta\right\rangle\right\rangle$ versus $\delta_{2}$ and $t$ when the two electric field components have the same weight $\gamma=0.5$ and by including progressively the three interactions with the two-color electric field in the Hamiltonian. For all considered configurations, the $t_{0}$-averaged alignment satisfies the symmetry relation (20) for $k=2, n_{1}=0$ and $n_{2}=1$. For $H=H_{0}+H_{\mu},\left\langle\left\langle\cos ^{2} \theta\right\rangle\right\rangle$ depends very weakly on $\delta_{2}$, and oscillates as $t$ increases quasi-periodically between 0.3 and 0.6 , see Fig. 8 (a). By also taking into account the interaction with the molecular polarizability and with the hyperpolarizability, $\left\langle\left\langle\cos ^{2} \theta\right\rangle\right\rangle$ show a rather weak dependence on $\delta_{2}$ for short propagation times, which becomes stronger for $t \gtrsim 200 \mathrm{ps}$, see Figs. 8 (b) and (c). In these cases, the oscillations of $\left\langle\left\langle\cos ^{2} \theta\right\rangle\right\rangle$ versus $t$ are faster and reach up to 0.9 , indicating that the molecule is strongly aligned.

The analytic expression (A9) provides the dependence on $\delta_{2}$ of the expectation values $\left\langle\left\langle\cos ^{2} \theta\right\rangle\right\rangle$. In Fig. 9, $\left\langle\left\langle\cos ^{2} \theta\right\rangle\right\rangle$ is plotted as a function of the second-harmonic phase for $\gamma=0.5$ and propagation times $t=200 \mathrm{ps}$ and $t=600 \mathrm{ps}$. The fitted coefficients $\mathcal{C}_{j}\left(t, \epsilon_{1}, \epsilon_{2}\right)$ and phases $\varphi_{j}\left(t, \epsilon_{1}, \epsilon_{2}\right)$, with $j$ being an even integer, of these numerical results to the series (A9) are presented in Fig. 9 (c-d), and (e-f), respectively. When only the electric field interaction with the electric dipole moment is taken into account, the alignment does not depend on $\delta_{2}$, and the $j=0$ coefficient is enough to accurately reproduce this result, i. e., $\mathcal{C}_{0}\left(t, \epsilon_{1}, \epsilon_{2}\right)$ is only the fitting parameter. The next term $\mathcal{C}_{2}\left(t, \epsilon_{1}, \epsilon_{2}\right)$ is smaller than $2 \times 10^{-4}$. For $H=H_{0}+H_{\mu}+H_{\alpha}$, higher order terms are needed in the $\left\langle\left\langle\cos ^{2} \theta\right\rangle\right\rangle$ analytical expansion, and they become smaller than $10^{-4}$ for $j \geq 6$ and $j \geq 8$ for $t=200 \mathrm{ps}$ and $t=600 \mathrm{ps}$, respectively. By adding $H_{\beta}$, i. e., $H=H_{0}+H_{\mu}+H_{\alpha}+H_{\beta}$, the $\delta_{2}$-dependence of $\left\langle\left\langle\cos ^{2} \theta\right\rangle\right\rangle$ becomes more complex, and even higher order terms are required for an accurate fitting. For the fitting phases $\varphi_{j}\left(t, \epsilon_{1}, \epsilon_{2}\right)$ in these two cases, a broad range of values is encountered, see Figs. 9 (e) and (f).

To conclude, the dependence of $\left\langle\left\langle\cos ^{2} \theta\right\rangle\right\rangle$ on $\gamma$ and $t$ is illustrated in Fig. 10 for $\delta_{2}=3 \pi / 4$. If only the electric field interaction with the electric dipole moment is taken into account, see Fig. 10 (a), the $t_{0}$-alignment oscillates between 0.3 and 0.7 , and reaches the largest values in the region $\gamma<0.5$, i. e., when the field strength of the firstharmonic is bigger than the one of the second-harmonic. For fixed $\gamma,\left\langle\left\langle\cos ^{2} \theta\right\rangle\right\rangle$ shows quite regular oscillations versus time. By taking into account the electric field interaction with the polarizability and with both polarizability and hyperpolarizability, Fig. 10 (b) and Fig. 10 (c) respectively, $\left\langle\left\langle\cos ^{2} \theta\right\rangle\right\rangle$ reaches larger values, up to 0.9 , and the frequency of its oscillations is increased.

\section{CONCLUSIONS}

The impact of a two-color continuous-wave nonresonant laser field in the rotational dynamics of a linear polar molecule has been investigated. Working within the Born-Oppenheimer and the rigid-rotor approximations, the symmetries of the Hamiltonian have been explored and their effects on the field-dressed rotational dynamics. Due to these symmetries, it is not possible to orient the polar molecule with a one-color cw laser field being necessary to employ a two-color one having odd and even products of the laser frequency.

The validity of the time-average approximation is first investigated assuming that this cw-non-resonant field does not drive any electronic, vibrational or rotational transitions, and despite that the laser field is not a pulse as normally used in experiments. For a cw-laser, this approximation starts to fail for laser periods satisfying $T \gtrsim 10 \mathrm{fs}$. The field-dressed rotational dynamics has been analyzed in the regime where the time-average approximation is not correct. By systematically including the interaction of the field with the electric dipole moment, polarizability and hyperpolarizability in the Hamiltonian, the $t_{0}$-averaged orientation and alignment have been analyzed versus the two-color laser field phase and relative strength of the two components. If only the interaction with the electric dipole moment is taken into account, the orientation has sinusoidal dependence on this relative phase, whereas the alignment is independent of it. Being possible to reproduce these numerical results with single-term analytical expressions. By considering the interaction with 

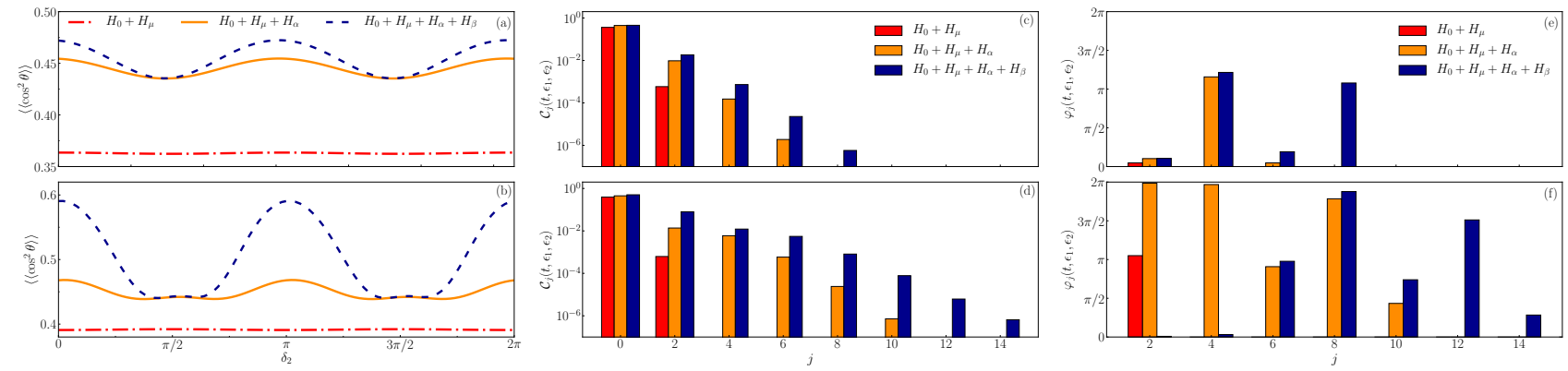

FIG. 9. For a two-color electric field with $\gamma=0.5, t_{0}$-averaged alignment as a function of the second-harmonic phase at fixed propagation times (a) $t=200 \mathrm{ps}$ and (b) $t=600 \mathrm{ps}$. (c) and (d) fitted coefficients $\mathcal{C}_{j}\left(t, \epsilon_{1}, \epsilon_{2}\right)$, and (e) and (f) fitted phases $\varphi_{j}\left(t, \epsilon_{1} \epsilon_{2}\right)$ from the analytic expression (A9) of $\left\langle\left\langle\cos ^{2} \theta\right\rangle\right\rangle$ for $t=200 \mathrm{ps}$ and $t=600$ ps, respectively. The interaction Hamiltonian includes $H_{\mu}$ (red dot-dashed line), $H_{\mu}+H_{\alpha}$ (orange solid line), and $H_{\mu}+H_{\alpha}+H_{\beta}$ (blue dashed line).

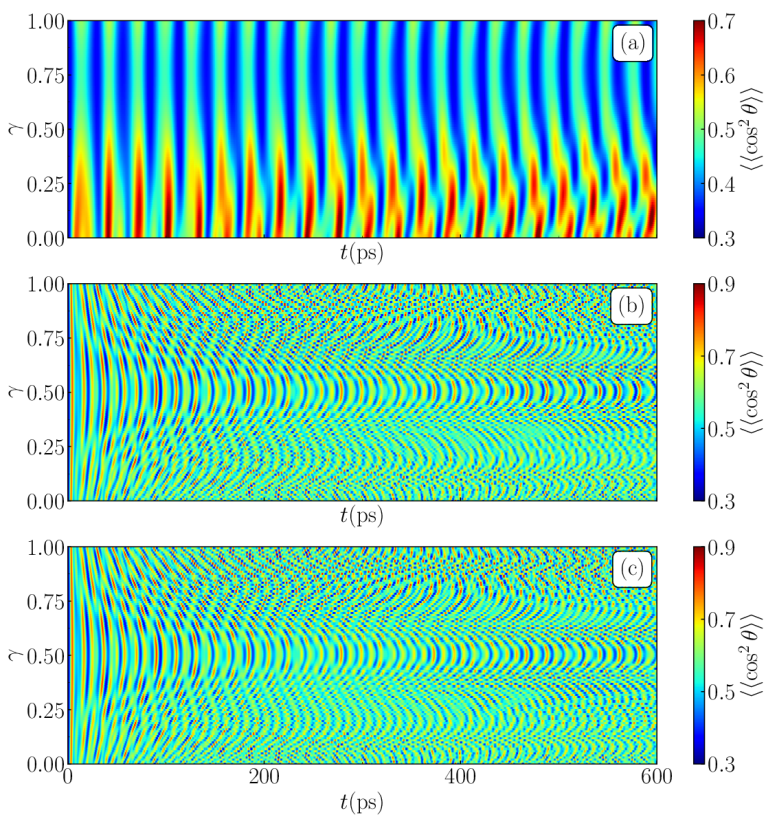

FIG. 10. The $t_{0}$-averaged alignment as a function of the propagation time and the relative weight of the electric field components $\gamma$ for the second-harmonic phase $\delta_{2}=3 \pi / 4$. The interaction Hamiltonian includes (a) $H_{\mu}$, (b) $H_{\mu}+H_{\alpha}$, and (c) $H_{\mu}+H_{\alpha}+H_{\beta}$.

the molecular polarizability, and, in addition with the hyperpolarizability, the orientation and alignment show a more complex, but still symmetric, dependence on the phase. As a consequence, more terms on the analytical expression are required to properly reproduce the numerical results. Regarding their dependence on the relative weight of the two electric field components, the largest $t_{0}$-averaged orientation and alignment are not necessarily obtained when both have the same weight.

Although this study is restricted to the OCS molecule, the observed physical phenomena occur in other polar molecules. A natural extension to this work would be to consider a linear molecule in a two-color cw laser fields with the two electric field components having perpendic- ular polarizations [52]. In this field-configuration, the symmetries of the system are reduced, and the two field components tend to align and orient the molecule in different directions. In addition, more complex molecules, such as symmetric or asymmetric tops, in two-color cw non-resonant laser field could be also explored.

\section{ACKNOWLEDGMENTS}

N.R.Q. acknowledges the financial support from the Alexander von Humboldt Foundation. Financial support by the Spanish Project No. FIS2017-89349-P (MINECO), and by the Andalusian research group FQM-207 is gratefully acknowledged. This study has been partially financed by the Consejería de Conocimiento, Investigación y Universidad, Junta de Andalucía and European Regional Development Fund (ERDF), Ref. SOMM17/6105/UGR.

\section{Appendix A: Analytic expressions of the orientation and alignment}

Following the results of Ref. [17-19], the orientation and the alignment can be expressed in terms of the amplitude and phase, $\epsilon_{j}$ and $\delta_{j}$, of the $j$-harmonics $g_{j}\left(t, t_{0}, \epsilon_{j}, \delta_{j}\right)=$ $\epsilon_{j} \cos \left[q_{j} \omega\left(t+t_{0}\right)+\delta_{j}\right]$, with $j=1, \ldots, s$, appearing in the Hamiltonian (2) due to the interaction of the molecule with the two-color electric field (1). The frequencies, amplitudes, and phases of these harmonics are collected in Table I. The first two rows in Table I with $j=1$ and 2 provide the harmonics of the biharmonic field, which appear due to the interaction of the biharmonic electric field with the permanent electric dipole moment. By including also the interaction of the biharmonic electric field with the molecular polarizability, a term proportional to $E^{2}(t)$ appears, and the harmonics with $j=3, \ldots, 6$, see Table I, also contribute to the Hamiltonian. The cubic term $E^{3}(t)$ is due to interaction with the molecular hyperpolarizability, and is responsible for the harmonics with $7 \leq j \leq 14$ in Table I. 


\begin{tabular}{|c|c|c|c|}
\hline$j$ & $q_{j}$ & $\epsilon_{j}$ & $\delta_{j}$ \\
\hline \hline 1 & $q_{1}$ & $\epsilon_{1}$ & $\delta_{1}$ \\
\hline 2 & $q_{2}$ & $\epsilon_{2}$ & $\delta_{2}$ \\
\hline 3 & $2 q_{1}$ & $\epsilon_{1}^{2} / 2$ & $2 \delta_{1}$ \\
\hline 4 & $2 q_{2}$ & $\epsilon_{2}^{2} / 2$ & $2 \delta_{2}$ \\
\hline 5 & $q_{1}+q_{2}$ & $\epsilon_{1} \epsilon_{2}$ & $\delta_{1}+\delta_{2}$ \\
\hline 6 & $q_{2}-q_{1}$ & $\epsilon_{1} \epsilon_{2}$ & $\delta_{2}-\delta_{1}$ \\
\hline 7 & $q_{1}$ & $(3 / 2) \epsilon_{1} \epsilon_{2}^{2}+(3 / 4) \epsilon_{1}^{3}$ & $\delta_{1}$ \\
\hline 8 & $q_{2}$ & $(3 / 2) \epsilon_{1}^{2} \epsilon_{2}+(3 / 4) \epsilon_{2}^{3}$ & $\delta_{2}$ \\
\hline 9 & $3 q_{1}$ & $\epsilon_{1}^{3} / 4$ & $3 \delta_{1}$ \\
\hline 10 & $3 q_{2}$ & $\epsilon_{2}^{3} / 4$ & $3 \delta_{2}$ \\
\hline 11 & $q_{2}+2 q_{1}$ & $(3 / 4) \epsilon_{1}^{2} \epsilon_{2}$ & $\delta_{2}+2 \delta_{1}$ \\
\hline 12 & $q_{2}-2 q_{1}$ & $(3 / 4) \epsilon_{1}^{2} \epsilon_{2}$ & $\delta_{2}-2 \delta_{1}$ \\
\hline 13 & $2 q_{2}+q_{1}$ & $(3 / 4) \epsilon_{1} \epsilon_{2}^{2}$ & $2 \delta_{2}+\delta_{1}$ \\
\hline 14 & $2 q_{2}-q_{1}$ & $(3 / 4) \epsilon_{1} \epsilon_{2}^{2}$ & $2 \delta_{2}-\delta_{1}$ \\
\hline
\end{tabular}

TABLE I. Prefactor of the frequency $\omega, q_{j}$, amplitude, $\epsilon_{j}$ and phase, $\delta_{j}$, of the $j$-harmonics appearing in the Hamiltonian (2) due to the interaction of the molecule with the two-color electric field (1).

Based on simple symmetry considerations of the $s$ harmonic functions, see Ref. [18, 19], the orientation and the alignment can be expressed as

$$
\begin{aligned}
& \left\langle\cos ^{k} \theta\right\rangle\left(t, t_{0}, \boldsymbol{\epsilon}, \boldsymbol{\delta}\right)= \\
& \sum_{\boldsymbol{n} \in \mathbb{Z}^{s}} C_{\boldsymbol{n}}(t, \boldsymbol{\epsilon}) \prod_{j=1}^{s} \epsilon_{j}^{\left|n_{j}\right|} \cos \left[\boldsymbol{n} \cdot \boldsymbol{\delta}+\omega t_{0} \boldsymbol{n} \cdot \boldsymbol{q}+\Theta_{\boldsymbol{n}}(t, \boldsymbol{\epsilon})\right]
\end{aligned}
$$

where $\boldsymbol{q}=\left\{q_{1}, \cdots, q_{s}\right\}, \boldsymbol{\epsilon}=\left\{\epsilon_{1}, \cdots, \epsilon_{s}\right\}, \boldsymbol{\delta}=$ $\left\{\delta_{1}, \cdots, \delta_{s}\right\}$, and $C_{\boldsymbol{n}}(t, \boldsymbol{\epsilon})$ and $\Theta_{\boldsymbol{n}}(t, \boldsymbol{\epsilon})$ are both even functions of each $\epsilon_{j}$. The $t_{0}$-averaged expectation values satisfy [19]

$$
\begin{aligned}
& \left\langle\left\langle\cos ^{k} \theta\right\rangle\right\rangle(t, \boldsymbol{\epsilon}, \boldsymbol{\delta})= \\
& C_{\mathbf{0}}(t, \boldsymbol{\epsilon})+\sum_{\boldsymbol{n} \in \mathbb{S}} C_{\boldsymbol{n}}(t, \boldsymbol{\epsilon}) \prod_{j=1}^{s} \epsilon_{j}^{\left|n_{j}\right|} \cos \left[\boldsymbol{n} \cdot \boldsymbol{\delta}+\Theta_{\boldsymbol{n}}(t, \boldsymbol{\epsilon})\right],
\end{aligned}
$$

where

$$
\mathbb{S}=\left\{\boldsymbol{n} \in \mathbb{Z}^{s}: \boldsymbol{n} \cdot \boldsymbol{q}=0\right\}
$$

denotes the set of nonzero solutions of the Diophantine equation $\boldsymbol{n} \cdot \boldsymbol{q}=0$, whose leftmost nonzero component is positive [18].

If only the interaction of the electric field with the electric dipole moment is included in Hamiltonian (2), $s=$ 2 , and $\boldsymbol{q}=\left\{q_{1}, q_{2}\right\}, \boldsymbol{\epsilon}=\left\{\epsilon_{1}, \epsilon_{2}\right\}$, and $\boldsymbol{\delta}=\left\{\delta_{1}, \delta_{2}\right\}[17,18]$. The Diophantine equation is $n_{1} q_{1}+n_{2} q_{2}=0$. The $t_{0^{-}}$ averaged expectation value (12) can be written as

$$
\begin{aligned}
& \left\langle\left\langle\cos ^{k} \theta\right\rangle\right\rangle\left(t, \epsilon_{1}, \epsilon_{2}, \delta_{1}, \delta_{2}\right)= \\
& \sum_{j=0}^{+\infty}\left|C_{j}\left(t, \epsilon_{1}, \epsilon_{2}\right)\right|\left(\epsilon_{1}^{q_{2}} \epsilon_{2}^{q_{1}}\right)^{j} \cos \left[j \xi_{12}+\Theta_{j}\left(t, \epsilon_{1}, \epsilon_{2}\right)\right],
\end{aligned}
$$

where $\xi_{12}=\left(q_{1} \delta_{2}-q_{2} \delta_{1}\right)$ and $\Theta_{0}\left(t, \epsilon_{1}, \epsilon_{2}\right)=0$ [19]. Due to the inversion of the electric field direction symmetry (16), it holds that: i) the series (A4) includes only even terms for $k$ even if $q_{1}+q_{2}$ is odd, otherwise all the terms contribute; ii) Eq. (A4) includes only odd ones for $k$ odd if $q_{1}+q_{2}$ is odd; and iii) if $q_{1}+q_{2}$ is even, $\left\langle\left\langle\cos ^{k} \theta\right\rangle\right\rangle=0$ with $k$ odd, and for $k=1$, the molecule is not oriented.

By including also the interaction with the polarizability, six harmonics appear in the Hamiltonian, i. e., $s=6$ in the set (A3) of non-zero solutions of the Diophantine equation, which reads

$$
\left(n_{1}+2 n_{3}+n_{5}-n_{6}\right) q_{1}+\left(n_{2}+2 n_{4}+n_{5}+n_{6}\right) q_{2}=0,
$$

and $\mathbb{S}=\left\{n_{1}=-2 n_{3}-n_{5}+n_{6}-m q_{2}, n_{1}>0, n_{2}=\right.$ $\left.-2 n_{4}-n_{5}-n_{6}+m q_{1},\left(m, n_{3}, n_{4}, n_{5}, n_{6}\right) \in \mathbb{Z}^{5}\right\}$. As a consequence, $\boldsymbol{n} \cdot \boldsymbol{\delta}=m \xi_{12}$. Using this result, Eq. (A2) can be rewritten as

$$
\begin{aligned}
& \left\langle\left\langle\cos ^{k} \theta\right\rangle\right\rangle\left(t, \epsilon_{1}, \epsilon_{2}, \delta_{1}, \delta_{2}\right)=C_{\mathbf{0}}\left(t, \epsilon_{1}, \epsilon_{2}\right)+ \\
& \sum_{(\boldsymbol{n}, m) \in \mathbb{S}} C_{\boldsymbol{n}}\left(t, \epsilon_{1}, \epsilon_{2}\right) \epsilon_{1}^{\left|x_{\boldsymbol{n}}\right|} \epsilon_{2}^{\left|y_{\boldsymbol{n}}\right|} \cos \left[m \xi_{12}+\Theta_{\boldsymbol{n}}\left(t, \epsilon_{1}, \epsilon_{2}\right)\right]
\end{aligned}
$$

where $x_{\boldsymbol{n}}, y_{\boldsymbol{n}}$ and $m$ are integers determined not only by the solutions of the Diophantine equation, but also by the symmetries. Indeed, the symmetry (16) implies that $\left|x_{\boldsymbol{n}}\right|+\left|y_{\boldsymbol{n}}\right|$ has the same parity as $k$. Thus, this expectation value (A6) is rewritten as

$$
\begin{aligned}
& \left\langle\left\langle\cos ^{k} \theta\right\rangle\right\rangle\left(t, \epsilon_{1}, \epsilon_{2}, \delta_{1}, \delta_{2}\right)= \\
& \sum_{j=0}^{+\infty} \mathcal{C}_{j}\left(t, \epsilon_{1}, \epsilon_{2}\right) \cos \left[j \xi_{12}+\varphi_{j}\left(t, \epsilon_{1}, \epsilon_{2}\right)\right],
\end{aligned}
$$

with $\mathcal{C}_{j}\left(t,-\epsilon_{1},-\epsilon_{2}\right)=(-1)^{k} \mathcal{C}_{j}\left(t, \epsilon_{1}, \epsilon_{2}\right)$. Furthermore, due to the symmetry on the phases (15) two cases can be distinguished according to the parity of $q_{1}+q_{2}$. (i) If $q_{1}+q_{2}$ is an even integer, $q_{1}$ and $q_{2}$ are both odd integer numbers because $\operatorname{gcd}\left(q_{1}, q_{2}\right)=1$. As a consequence of the symmetry (15), the identity $\left\langle\left\langle\cos ^{k} \theta\right\rangle\right\rangle=(-1)^{k}\left\langle\left\langle\cos ^{k} \theta\right\rangle\right\rangle$ is obtained. Therefore, the expectation values is zero for $k$ odd and satisfies (A7) for $k$ even. (ii) If $q_{1}+q_{2}$ is an odd integer, $q_{1}$ and $q_{2}$ have different parity. Due to the symmetry (15), for odd or even values of $k$ in (A7), only odd or even terms contribute to the corresponding expansion, respectively, i. e.,

$$
\begin{aligned}
& \left\langle\left\langle\cos ^{2 k+1} \theta\right\rangle\right\rangle\left(t, \epsilon_{1}, \epsilon_{2}, \delta_{1}, \delta_{2}\right)= \\
& \sum_{\substack{j=1 \\
(j \text { odd })}}^{+\infty} \mathcal{C}_{j}\left(t, \epsilon_{1}, \epsilon_{2}\right) \cos \left[j \xi_{12}+\varphi_{j}\left(t, \epsilon_{1}, \epsilon_{2}\right)\right],
\end{aligned}
$$

and

$$
\begin{aligned}
& \left\langle\left\langle\cos ^{2 k} \theta\right\rangle\right\rangle\left(t, \epsilon_{1}, \epsilon_{2}, \delta_{1}, \delta_{2}\right)= \\
& \sum_{\substack{j=0 \\
(j \text { even })}}^{+\infty} \mathcal{C}_{j}\left(t, \epsilon_{1}, \epsilon_{2}\right) \cos \left[j \xi_{12}+\varphi_{j}\left(t, \epsilon_{1}, \epsilon_{2}\right)\right]
\end{aligned}
$$

When the interaction between the electric field and 
hyperpolarizability is also included in the Hamiltonian, $s=14$ in the set of non-zero solutions (A3) of the Diophantine equation, which reads $\left[n_{1}+2\left(n_{3}+n_{11}-n_{12}\right)+\right.$ $\left.n_{5}-n_{6}+n_{7}+3 n_{9}+n_{13}-n_{14}\right] q_{1}+\left[n_{2}+2\left(n_{4}+n_{13}+\right.\right.$ $\left.\left.n_{14}\right)+n_{5}+n_{6}+n_{8}+3 n_{10}+n_{11}+n_{12}\right] q_{2}=0$. Therefore, $\mathbb{S}=\left\{n_{1}=-2\left(n_{3}+n_{11}-n_{12}\right)-n_{5}+n_{6}-n_{7}-3 n_{9}-n_{13}+\right.$ $n_{14}-m q_{2}, n_{1}>0, n_{2}=-2\left(n_{4}+n_{13}+n_{14}\right)-n_{5}-n_{6}-n_{8}-$ $\left.3 n_{10}-n_{11}-n_{12}+m q_{1},\left(m, n_{3}, n_{4}, \cdots, n_{14}\right) \in \mathbb{Z}^{13}\right\}$. As a consequence, $\boldsymbol{n} \cdot \boldsymbol{\delta}=m \xi_{12}$ is also satisfied. In this case, expression (A6) is also obtained, and a similar symmetry analysis transforms it into the formulas (A8) and (A9) if $q_{1}+q_{2}$ is an odd integer. Finally, note that (A4) can also be rewritten as (A8) and (A9) if $q_{1}+q_{2}$ is an odd integer.
[1] S. Flach, O. Yevtushenko, and Y. Zolotaryuk, Phys. Rev. Lett. 84, 2358 (2000).

[2] P. Reimann, Phys. Rep. 361, 57 (2002).

[3] M. Schiavoni, L. Sánchez-Palencia, F. Renzoni, and G. Grynberg, Phys. Rev. Lett. 90, 094101 (2003).

[4] S. Ooi, S. Savel'ev, M. B. Gaifullin, T. Mochiku, K. Hirata, and F. Nori, Phys. Rev. Lett. 99, 207003 (2007).

[5] P. Hänggi and F. Marchesoni, Rev. Mod. Phys. 81, 387 (2009).

[6] Y. Zolotaryuk and M. M. Osmanov, Eur. Phys. J. B 79, 257 (2011).

[7] D. Cubero, V. Lebedev, and F. Renzoni, Phys. Rev. E 82, 041116 (2010).

[8] N. R. Quintero, R. Alvarez-Nodarse, and J. A. Cuesta, J. Phys. A: Math. Gen. 44, 425205 (2011).

[9] K. Seeger and V. Maurer, Solid State Commun. 27, 603 (1978).

[10] A. V. Ustinov, C. Coqui, A. Kemp, Y. Zolotaryuk, and M. Salerno, Phys. Rev. Lett. 93, 087001 (2004).

[11] R. Gommers, S. Bergamini, and F. Renzoni, Phys. Rev. Lett. 95, 073003 (2005).

[12] A. Engel, H. W. Müller, P. Reimann, and A. Jung, Phys. Rev. Lett. 91, 060602 (2003).

[13] T. Salger, S. Kling, T. Hecking, C. Geckeler, L. MoralesMolina, and M. Weitz, Science 326, 1241 (2009).

[14] M. Salerno and Y. Zolotaryuk, Phys. Rev. E 65, 056603 (2002).

[15] L. Morales-Molina, N. R. Quintero, F. G. Mertens, and A. Sánchez, Phys. Rev. Lett. 91, 234102 (2003).

[16] N. R. Quintero, Soliton Ratchets in sine-Gordon-Like Equations, edited by Cuevas-Maraver J., Kevrekidis P., Williams F. "The sine-Gordon Model and its Applications. Nonlinear Systems and Complexity", vol 10 (Springer, Cham, 2014).

[17] N. R. Quintero, J. A. Cuesta, and R. Alvarez-Nodarse, Phys. Rev. E 81, 030102 (2010).

[18] J. A. Cuesta, N. R. Quintero, and R. Alvarez-Nodarse, Phys. Rev. X 3, 041014 (2013).

[19] J. Casado-Pascual, J. A. Cuesta, N. R. Quintero, and R. Alvarez-Nodarse, Phys. Rev. E 91, 022905 (2015).

[20] B. Friedrich and D. R. Herschbach, Nature 353, 412 (1991).

[21] B. Friedrich and D. R. Herschbach, Z. Phys. D 18, 153 (1991).

[22] B. Friedrich, D. P. Pullman, and D. R. Herschbach, J. Phys. Chem. 95, 8118 (1991).

[23] H. Stapelfeldt and T. Seideman, Rev. Mod. Phys. 75, 543 (2003).

[24] H. J. Loesch and A. Remscheid, J. Chem. Phys. 93, 4779 (1990).

[25] J. Bulthuis, J. Miller, and H. J. Loesch, J. Phys. Chem. A 101, 7684 (1997).
[26] H. Li, K. J. Franks, R. J. Hanson, and W. Kong, J. Phys. Chem. A 102, 8084 (1998).

[27] B. Friedrich and D. R. Herschbach, J. Chem. Phys. 111, 6157 (1999).

[28] B. Friedrich and D. Herschbach, J. Phys. Chem. A 103, 10280 (1999).

[29] H. Sakai, S. Minemoto, H. Nanjo, H. Tanji, and T. Suzuki, Phys. Rev. Lett. 90, 083001 (2003).

[30] S. Minemoto, H. Nanjo, H. Tanji, T. Suzuki, and H. Saka, J. Chem. Phys. 118, 4052 (2003).

[31] L. Holmegaard, J. H. Nielsen, I. Nevo, H. Stapelfeldt, F. Filsinger, J. Küpper, and G. Meijer, Phys. Rev. Lett. 102, 023001 (2009).

[32] J. H. Nielsen, H. Stapelfeldt, J. Küpper, B. Friedrich, J. J. Omiste, and R. González-Férez, Phys. Rev. Lett. 108, 193001 (2012).

[33] J. J. Omiste and R. González-Férez, Phys. Rev. A 86, 043437 (2012).

[34] C.-C. Shu, K.-J. Yuan, W.-H. Hu, and S.-L. Con, J. Chem. Phys. 132, 244311 (2010).

[35] S. Fleischer, Y. Zhou, R. W. Field, and K. A. Nelson, Phys. Rev. Lett. 107, 163603 (2011).

[36] K. Kitano, N. Ishii, and J. Itatani, Phys. Rev. A 84, 053408 (2011).

[37] M. Lapert and D. Sugny, Phys. Rev. A 85, 063418 (2012).

[38] C.-C. Shu and N. E. Henriksen, Phys. Rev. A 87, 013408 (2013).

[39] K. N. Egodapitiya, S. Li, and R. R. Jones, Phys. Rev. Lett. 112, 103002 (2014).

[40] T. Kanai and H. Sakai, J. Chem. Phys. 115, 5492 (2001).

[41] K. Oda, M. Hita, S. Minemoto, and H. Sakai, Phys. Rev. Lett. 104, 213901 (2010).

[42] P. M. Kraus, D. Baykusheva, and H. J. Wörner, J. Phys. B: At. Mol. Opt. Phys. 47, 124030 (2014).

[43] P. M. Kraus, D. Baykusheva, and H. J. Wörner, Phys. Rev. Lett. 113, 023001 (2014).

[44] D. Baykusheva, M. S. Ahsan, N. Lin, and H. J. Wörner, Phys. Rev. Lett. 116, 123001 (2016).

[45] P. S. Pershan, J. P. van der Ziel, and L. D. Malmstrom, Phys. Rev. 143, 574 (1966).

[46] J. O. Hirschfelder, Intermolecular Forces (Interscience Publishers, 1967).

[47] A. J. Stone, The Theory of Intermolecular Forces (Clarendon Press, 1996).

[48] M. Beck, A. Jäckle, G. Worth, and H. D. Meyer, Phys. Rep. 324, 1 (2000).

[49] G. Maroulis and M. Menadakis, Chem. Phys. Lett. 494, 144 (2010).

[50] B. Deppe, G. Huber, C. Kränkel, and J. Küpper, Opt. Express 23, 28491 (2015).

[51] S. De, I. Znakovskaya, D. Ray, F. Anis, N. G. Johnson, I. A. Bocharova, M. Magrakvelidze, B. D. Esry, C. L. 
Cocke, I. V. Litvinyuk, and M. F. Kling, Phys. Rev. Lett. 103, 153002 (2009).
[52] J. H. Mun, H. Sakai, and R. González-Férez, Phys. Rev. A 99, 053424 (2019). 